Revue d'histoire de l'enfance « irrégulière »

Le Temps de l'histoire

Hors-série | 2001

Histoire et justice, panorama de la recherche

\title{
La justice royale de Montferand (1425-1455)
}

Josiane Teyssot

\section{Q OpenEdition}

Journals

Édition électronique

URL : http://journals.openedition.org/rhei/445

DOI : 10.4000/rhei.445

ISBN : 978-2-7535-1641-0

ISSN : 1777-540X

Éditeur

Presses universitaires de Rennes

Édition imprimée

Date de publication : 15 novembre 2001

Pagination : 173-179

ISSN : 1287-2431

Référence électronique

Josiane Teyssot, "La justice royale de Montferand (1425-1455) », Revue d'histoire de l'enfance " irrégulière » [En ligne], Hors-série | 2001, mis en ligne le 31 mai 2007, consulté le 21 avril 2019. URL http://journals.openedition.org/rhei/445; DOI : 10.4000/rhei.445 


\section{La justice royale de Montferrand (1425-1455). Mise en place, organisation, fonctionnement}

La présente contribution découle de deux sources principales : André Bossuat, Le bailliage royal de Montferrand (1425-1556), publié en 1957, et toujours irremplaçable, et Éric Braure, Le bailliage royal de Montferrand, d'après ses registres d'assises de 1425 à 1456, mémoire de maîtrise, Lyon III, 1999. Le premier voit surtout la mise en place de ce bailliage, sans ressort à l'origine, et ses fins politiques dans le cadre de l'État monarchique en construction : il établit la liste des principaux officiers. Le second étudie le bailliage dans son fonctionnement matériel et judiciaire, dans les trente premières années de son existence, c'est-à-dire quand le bailli est Hervé du Mesnil. C'est ainsi qu'apparaissent les acteurs subalternes, mais essentiels, comme les sergents, et que l'on assiste à l'implantation au quotidien de cette nouvelle structure judiciaire.

Ces travaux sont complétés par mes propres recherches sur les villes d'Auvergne à la fin du Moyen Âge, et en tout premier lieu, sur les trois agglomérations principales : Riom, Montferrand, Clermont. ${ }^{(2)}$

Montferrand abrite à partir de 1425, et jusqu'en 1556, un bailliage particulier comme il en existe quelques autres autour ou dans les dernières grandes principautés non encore rattachées au Domaine royal. Il y a par exemple Saint-Pierre-le-Moûtier au nord du duché de Bourbonnais (avec sa lieutenance de Cusset), et Mâcon au sud du duché de Bourgogne. Ces bailliages sont d'abord des sièges de justice royale avant d'être également des bastions politiques du pouvoir royal qui empiètent sur les prérogatives des derniers grands féodaux.

Pour bien comprendre le fonctionnement de celui de Montferrand, il convient de retracer dans un premier temps les grandes lignes du contexte régional : ce contexte est primordial pour la compréhension
Josiane

Teyssot $^{(1)}$

(1) Maître de conférences d'histoire médiévale, université Blaise Pascal, ClermontFerrand II.

(2) Josiane TEYSSOT, Riom, capitale et bonne ville d'Auvergne, 12121557, Nonette, éditions Créer, 1999, 432 p., et Josiane TEYSSOT, "Le réseau urbain de Basse Auvergne du XIIIème au XVIème siècle”, Villes moyennes : espace, société, patrimoine, Lyon, PUL, 1997, pp. 127-133. Pour

Josiane Teyssot / pp. 173 à 179 
l'ouvrage d'Éric BRAU-

$\mathrm{RE}$, seules les grandes

lignes sont données ici, avec l'autorisation de l'auteur. Ce mémoire de maîtrise, que j'ai eu le plaisir de diriger, doit en effet se poursuivre par un travail de troisième cycle : ses schémas et ses cartes ne sont donc pas reproduits ici. du sujet. Viendront ensuite l'organisation et le fonctionnement de ce bailliage.

\section{Le contexte de la mise en place :}

l'Auvergne au début du XVème siècle.

Depuis les traités d'Azay-le-Rideau et de Gisors en 1189, l'Auvergne relève officiellement de la mouvance capétienne, alors qu'auparavant, elle se rattachait à l'Aquitaine des Plantagenêts. Mais certains seigneurs locaux, dont le comte, demeurent réticents à ce changement. En 1212, l'armée royale de Philippe Auguste s'empare donc par la force d'une grande partie du comté, c'est-à-dire de la vallée de l'Allier avec la grande route nord-sud dite des Français. Cette nouvelle possession est appelée la Terre royale d'Auvergne. Elle est confiée en apanage, de 1241 à 1271, à Alphonse de Poitiers, frère de Saint-Louis, puis elle retourne à la Couronne en 1271. Ces bouleversements politiques sont apparemment bien acceptés par les habitants car ils favorisent la paix et la stabilité dans la région, contrairement aux événements des siècles précédents. La prospérité aide également. Cette situation s'inverse quand intervient le deuxième apanage, celui de Jean, duc de Berry et d'Auvergne.

Ce dernier apanage est essentiel. Jean de Berry, fils du roi Jean II le Bon, frère de Charles V, oncle de Charles VI, est un prince très puissant et très influent en ce début du XVème siècle. Mais il a perdu ses deux fils prématurément, en 1383 et 1397 : seule reste sa fille, Marie de Berry, mariée en 1400 à Jean, duc de Bourbonnais. Or, la règle de l'apanage veut qu'il ne puisse pas y avoir de succession par les femmes. Pour éviter cet écueil, le contrat de mariage de Marie, en mai 1400, accepté par le roi Charles VI, stipule que la fille de Jean de Berry gardera une partie de l'apanage de son père, en l'occurrence l'Auvergne, à condition que son époux, le duc de Bourbonnais, reconnaisse que toutes ses terres plus l'Auvergne constituent elles-mêmes un apanage. La Couronne pourra éventuellement récupérer cet apanage Bourbonnais-Auvergne en cas de non-descendance mâle dans l'avenir.

Quand le vieux duc de Berry meurt en juin 1416, sa succession semble donc réglée. En fait, il n'en est rien à cause des troubles et des diffi- 
cultés que traverse le royaume de Charles VI le Fou. D'abord, la guerre de Cent Ans a repris : le 25 octobre 1415, c'est la déroute française d'Azincourt. La noblesse du royaume est massacrée, le roi d'Angleterre Henri $\mathrm{V}$ ne faisant que deux prisonniers : les ducs d'Orléans et de Bourbonnais. Ensuite, la guerre civile entre Armagnacs et Bourguignons fait rage à partir de 1411 : le vieux duc de Berry et son gendre le duc de Bourbonnais (avant sa capture en 1415) sont dans le camp armagnac. Or, en 1416, les Armagnacs n'ont pas le dessus : ils ont repris Paris aux Bourguignons après les excès de la révolte cabochienne de 1413, avant de perdre à nouveau la capitale en 1418 par suite de leurs propres excès. En conséquence, les gens du Parlement de Paris, soucieux des intérêts de la monarchie, même défaillante, refusent d'entériner la succession telle qu'elle était prévue dans le contrat de mariage de 1400. Ce n'est qu'en 1425 que le petit roi de Bourges, Charles VII, qui se trouve vraiment au plus bas, politiquement et militairement, accepte de valider la succession car il a besoin du soutien du clan bourbonnais, celui-ci étant mené par la duchesse Marie et son jeune fils Charles (Jean de Bourbonnais reste prisonnier en Angleterre jusqu'à sa mort en 1434, sans revoir la France).

En 1425 donc, après neuf ans d'incertitude, l'Auvergne est intégrée à l'apanage bourbonnais, hormis la ville de Montferrand. Le roi argue en effet des privilèges de la ville accordés par Louis VIII en 1225 pour la distraire du reste de la province. ${ }^{(3)}$

Telles sont donc les conditions de mise en place de ce bailliage spécial, sans ressort autre que celui assez restreint de la châtellenie (ou ancienne prévôté) de Montferrand à l'origine, et avec pour seuls justiciables officiels les exempts de Basse Auvergne, c'est-à-dire les sujets qui relèvent directement de la justice royale, comme les grandes institutions ecclésiastiques de la région.

\section{L'organisation du bailliage.}

Un bailliage normal est une circonscription administrative et judiciaire du Domaine royal : le bailli gère les biens du roi, il réunit l'ost en cas de besoin, il veille à la bonne levée des impôts et des taxes, et il rend la justice au nom du souverain en première instance. Il peut aussi tenir
(3) En 1225, Louis

VIII place les

Montferrandais sous sa

protection, et il avantage

leurs foires et marchés

en échange du paiement

annuel d'un impôt

supplémentaire équiva-

lant à un marc d'or, soit

$244 \mathrm{~g}$ de métal précieux.

Il promet de ne jamais

soustraire la ville au

Domaine royal, ce qui

est pourtant fait en 1360

au profit de Jean de

Berry, malgré les vives

protestations des habi-

tants. Josiane TEYSSOT,

"Le marc d'or de

Montferrand", Bulletin

bistorique et scientifique de

l'Auvergne, 1999, nº 741,

pp. 65-73. 
(4) Jean-François

LEMARIGNIER, $\mathrm{La}$

France médiévale : institu-

tions et société, Paris,

Armand Colin, 1970,

pp. 338-345. Albert

RIGAUDIÈRE, Pouvoirs

et institutions dans la

France médiévale. Des

temps féodaux aux temps

de l'État (tome II), Paris,

Armand Colin, 1994,

pp. $255-259$

(5) BOSSUAT, op. cit., pp. 103-125. BRAURE, op. cit., pp. 47-49 et pp. 61-73. une première justice d'appel en cas de litige concernant une justice seigneuriale vassale du roi de France, le Parlement de Paris restant en ce cas l'ultime juridiction d'appel.(4)

Pour un bailliage spécial comme celui de Montferrand on peut parler de "bailliage croupion" ; qu'en est-il ? Ce sont les fonctions judiciaires qui constituent l'essentiel des prérogatives bailliagères. En ce cas, le bailli est avant tout un homme de loi, entouré d'hommes de loi, du moins en théorie puisque le premier bailli, Hervé du Mesnil, n’est pas un homme de loi mais un fidèle du roi.

L'organigramme du bailliage est donc le suivant :

- un bailli,

- un lieutenant général (dès 1426),

- deux lieutenants particuliers (un en 1426, le second à partir de 1455),

- un procureur du roi,

- un substitut du procureur,

- un avocat du roi.

Viennent ensuite un greffier, un receveur, un garde du sceau, un châtelain (chargé de la police dans la ville même de Montferrand) et son lieutenant, un procureur de la châtellenie et un prévôt des exempts (à partir de 1490 seulement). Enfin, il y a les sergents recrutés localement pour faire appliquer les décisions prises par le bailli ou ses adjoints.

En ce qui concerne les officiers principaux, le lieutenant général remplace le bailli dans la plénitude de ses pouvoirs quand le bailli est absent : par exemple, s'il est appelé auprès du roi pour rendre des comptes. Les lieutenants particuliers se partagent, eux, le droit civil et le droit criminel, tandis que le procureur du roi est chargé de l'accusation au nom du roi, ainsi que son adjoint le substitut. L'avocat du roi défend quant à lui les intérêts particuliers du souverain dans le ressort du bailliage. ${ }^{(5)}$

Tous ces officiers du bailliage, du plus important comme le bailli, au plus petit comme le sergent, sont nommés par le roi et ils prêtent un serment de fidélité au souverain. Le bailli est choisi, au début, à l'extérieur de la région. Hervé du Mesnil, le premier bailli de Montferrand, celui qui a 
installé l'institution, et finalement, lui a donné tout son poids car il est resté en place 30 ans, est de petite noblesse d'Ile-de-France ou de Champagne. ${ }^{(6)}$ Sa mère, Jeanne, était demoiselle d'honneur du roi Charles VII et avait été la "gouverneresse" du jeune roi (elle l'avait déniaisé) : elle entre ensuite au service de la reine Marie d'Anjou. Elle a deux fils : Jean, conseiller et chambellan du roi, et Hervé qui est premier panetier dès 1420, puis premier maître d'hôtel du roi, avant d'être nommé bailli de Montferrand. Hervé du Mesnil appartient donc à ces fonctionnaires subalternes qui doivent tout à la royauté et qui lui sont totalement dévoués, au point qu'ils contribuent largement à sauver l'État monarchique gravement menacé par la guerre civile entre Armagnacs et Bourguignons.

Les autres officiers sont de recrutement local, sans doute sur proposition du bailli. Il faut cependant excepter le premier lieutenant général Guillaume Toulouzain, probablement pas Auvergnat, qui reste en place jusqu'en 1458, c'est-à-dire après la mort d'Hervé du Mesnil. Cette longévité dans la fonction est elle aussi exceptionnelle : le roi laisse longtemps en place ces deux premiers officiers qui lui donnent entière satisfaction dans l'implantation réelle du bailliage.

Les recrutements "locaux" ne sont pas de Montferrand même la plupart du temps : ils viennent de toute la province comme Étienne Ébrard, lieutenant général de 1458 à 1462, dont la famille est originaire d'Aigueperse après une implantation temporaire à Riom. ${ }^{(7)}$ Le bailliage de Montferrand devient un débouché majeur pour les carrières des juristes locaux, à côté de Riom, siège de la justice et des tabellions ducaux.

En résumé, le roi impose ses hommes, surtout au début du bailliage, en installant des fidèles venus d'ailleurs. Quand l'institution est solidement implantée, il recrute sur place, y compris le bailli, car le pouvoir royal n'est plus contesté, notamment au XVIème siècle, quand le duché de Bourbonnais est rattaché à la Couronne en 1523.

\section{Le fonctionnement du bailliage sous Hervé du Mesnil.}

C'est la période de fonctionnement la plus intéressante sur le plan politique car elle constitue la base de la réussite future du bailliage.

En effet, le dépouillement des registres d'assises fournit des informa-
(6) Cela change au début du XVIème siècle avec François de

Chazeron, bailli de 1508 à 1524, et avec Antoine de Chazeron, bailli de 1525 à 1556 : tous les deux appartiennent à un petit et ancien lignage noble d'Auvergne. Ils sont les seuls, avec Hervé du Mesnil, à rester aussi longtemps en place : les autres titulaires siègent 2 à 13 ans. Parmi eux figure le conseiller de Louis XI, Jean de Doyat, de 1480 à 1481 .

(7) Josiane TEYSSOT, Riom, capitale..., op. cit., pp. 187-188. 
(8) BRAURE, op. cit., cartes pp. 57-59 et tableau p. 56.

(9) Cette lieutenance d'Usson est directement exercée par le bailli de Montferrand qui cumule ainsi les deux fonctions. BRAURE, op. cit., carte p. 106. La famille comtale d'Auvergne s'est scindée en deux branches au milieu du XIIème siècle. Dans des conditions mal connues, Guillaume VII le Jeune a été spolié par son oncle Guillaume le Vieux. Ce dernier l'a emporté : il est à l'origine des comtes d'Auvergne, ceux-là même qui sont vaincus par le roi en 1212.

Guillaume le Jeune n'a conservé que les terres pauvres des plateaux occidentaux de la province, plus Montferrand et des droits très théoriques sur Clermont : cette branche, aînée à l'origine, est ainsi devenue secondaire par rapport aux comtes, et elle tions précieuses même si ces registres ne détaillent pas la plupart du temps le fonds des affaires traitées : les parties sont citées et les condamnations ou renvois sont mentionnées, mais pas toujours. Ces maigres données permettent cependant de cerner la "tactique" du bailli : se mêler de la justice des vassaux voisins, avec l'approbation de la population qui comprend tout l'intérêt pour elle de cette rivalité des pouvoirs, puis rendre un verdict le plus juste possible même si c'est le plus souvent en défaveur du seigneur local, et prendre ensuite prétexte de ce premier exemple pour considérer toute la seigneurie en question comme relevant dorénavant de la juridiction bailliagère. C'est le meilleur moyen d'étendre, et même de créer, la circonscription du bailliage, qui au départ n'existait pratiquement pas. C'est ainsi qu'apparaît une première zone d'extension, essentiellement vers le sud et l'ouest, d'un rayon d'environ $18 \mathrm{~km}$, puis ce sont des zones de plus en plus larges : $35 \mathrm{~km}, 47,5 \mathrm{~km}, 54 \mathrm{~km}$. Il s'agit d'une avancée méthodique renforcée en parallèle par une multiplication du nombre des sergents : il ne sert à rien de multiplier les causes et les procès traités s'il n'y a pas un bras armé efficace pour concrétiser, très matériellement, les avancées ainsi réalisées. On passe ainsi de 8 sergents en 1426 à 52 en $1456{ }^{\left({ }^{(8)}\right.}$

Cette avancée s'opère au détriment des seigneurs secondaires de la province comme le Dauphin d'Auvergne, branche des anciens comtes. Quand meurt Hervé du Mesnil, tout le Dauphiné est passé sous la tutelle du bailliage royal de Montferrand, ou de sa lieutenance d'Usson. ${ }^{(9)}$

Cependant, le duc lui aussi pâtit du voisinage : d'abord dans sa seigneurie de Thiers, ancien siège d'une prévôté royale, et que lui dispute le roi, mais cette question reste encore mal étudiée. Il existe également quelques cas de litiges dans la région de Riom, comme le problème des pâturages entre Riom et le village de St-Bonnet juste à côté : en 1452, les bourgeois riomois décident d'empêcher la vaine pâture des petits paysans, dont ceux de St-Bonnet, pour se livrer à l'élevage spéculatif, notamment de moutons. Le bailli s'en est mêlé, a donné raison aux habitants de Saint-Bonnet, ce qui a ensuite été confirmé par les Grands Jours royaux. ${ }^{(10)}$ Mais en 1469 , le sénéchal ducal, le duc et son conseil, après s'être déplacés sur le site, favorisent les Riomois. Le bailli de 
Montferrand d'alors, Louis de Beaufort, fait comparaître les parties, sans tenir compte de l'arrêt ducal, en arguant qu'un cas déjà passé devant la juridiction royale ne peut plus être traité par le tribunal ducal. Le Parlement de Paris entérine cette vision des choses. ${ }^{(11)}$

Mais Hervé du Mesnil "rogne" aussi les pouvoirs urbains. En effet, depuis le milieu du XIVème siècle, les treize bonnes villes de Basse Auvergne jouent un rôle politique important à travers les États provinciaux, très dynamiques, qui ont assuré l'essentiel de la défense de la province lors de la guerre de Cent Ans. Le bailli intervient notamment à Montferrand en profitant là aussi de la question des pâturages : les gros marchands, dont les affaires commerciales périclitent, se reconvertissent dans l'élevage spéculatif pour nourrir les armées, en particulier les Ecorcheurs du duc. Ils interdisent la vaine pâture, et le bailli intervient pour faire respecter la coutume d'Auvergne, et en même temps pour préserver les intérêts militaires de la Couronne. La querelle s'envenime et débouche finalement sur une crise consulaire : les hommes de loi réclament de participer au pouvoir communal et Hervé du Mesnil les encourage, pour casser l'assise de l'oligarchie marchande qui accapare le consulat et fournit l'armée ducale. C'est chose faite en 1447. A la même date, le duc réforme lui aussi le consulat de Riom, ce qui n'est certainement pas une simple coïncidence. ${ }^{(12)}$

Voilà donc les grandes lignes du fonctionnement d'une justice royale particulière au XVème siècle, à partir d'un bailliage "croupion" très efficace juridiquement et surtout politiquement. On connaissait l'avancée du pouvoir royal au XIIIème siècle par l'intermédiaire de sa justice d'appel, avec la création du Parlement de Paris en 1254, mais cela continue donc après, à l'aube des Temps Modernes, quand l'État monarchique se met véritablement en place.

Deux autres domaines jouent le même rôle, tout aussi efficacement, pendant les trois derniers siècles du Moyen Âge : la monnaie et le sceau authentique, c'est-à-dire le notariat qui garantit les actes privés. ${ }^{(13)}$ Mais ces points restent encore largement à étudier. a pris le nom de

Dauphins d'Auvergne par suite du mariage de Guillaume le Jeune avec Dauphine, fille du comte de Vienne (BALUZE, Histoire généalogique de la maison d'Auvergne, 1708, tome I, pp. 61-65).

(10) Il s'agit sans doute des Grands Jours de 1454-1455 tenus à Montferrand, mais dont il ne subsiste malheureusement pas d'archives.

(11) Josiane TEYSSOT, Riom, capitale..., op. cit., pp. 204-206.

(12) Josiane TEYSSOT, "Une crise urbaine au milieu du XVème siècle : Montferrand, 1442-1447”, Bulletin historique et scientifique de l'Auvergne, 1991, n 708, pp. 195-203.

(13) En effet, une ordonnance royale de 1263 impose la circulation des espèces royales dans tout le royaume contrairement aux monnaies féodales. Ces der- 